\title{
Regularized image restoration based on preconditioned conjugate gradient method
}

\author{
Ling Meng ${ }^{1}$ and Bing Zhang ${ }^{2}$
}

\begin{abstract}
Image restoration can be attributed to solving a linear systems and conjugate gradient method is an effective iteration algorithm for solving various linear systems. However the convergence rate of CG method is determined by condition number of coefficient matrix. The level1 and level2 preconditioner were used to reduce the condition number of coefficient matrix and to accelerate the convergence rate. Simulation experiment show that with same iteration times the PCG method reached better results than other method.
\end{abstract}

Keywords Image restoration, Preconditioned matrix, Conjugate gradient method, Toeplitz matrices, Preconditioned conjugate gradient method.

\section{Introduction}

The discrete image degradation model can be described as the linear systems as follows(see [2,9])

$\operatorname{vec}(d)=T \square \operatorname{vec}(f)+\operatorname{vec}(\eta)$

where $T$ is a block Toeplitz matrix with Toeplitz blocks, vec $(d), \operatorname{vec}(g)$ and $\operatorname{vec}(\eta)$ are vectors which the discrete blurred image $d$, discrete original image $f$ and noise $\eta$ are reordered with column lexicographical order respectively. To

\author{
1..L.Meng \\ School of science, Communication University of China, Beijing. \\ e-mail: mengling@cuc.edu.cn \\ 2.B. Zhang $(\bowtie)$ \\ School of science, Communication University of China, Beijing. \\ e-mail:zhangbin628@cuc.edu.cn
}


solve this ill-posed linear systems, we use the Tikhonov regularization method and obtain the following systems

$$
\left(T^{*} T+\alpha L\right) f=T^{*} d
$$

where $L$ is symmetric positive definite block matrix. $\alpha>0$ is the regularization parameter. To solve linear systems(2) is equivalent to solve the minimum value of the quadratic function :

$$
\min _{f} J(f)=\frac{1}{2} f^{T} A f+b^{T} f+c, A=T^{*} T+\alpha L, b=T^{*} d
$$

The minimization of quadratic function was solved by conjugate gradient(CG) method [1] in optimization theory [2].The convergence rate of this iteration method is determined by condition number of $A$. The distribution of singular value of $A$ is moer concentrated, the condition number of $A$ is smaller and the convergence rate is fast. Preconditioned conjugate gradient (PCG) method $[1,3]$ is an improved algothm for the CG method. We introduced the preconditioner $M$ first to enable $M^{-1} A$ has more concentrated singular value distribution, then substituted $A$ with $M^{-1} A$ in iteration algothm to accelerate the convergence rate.

\section{Block Circulant Preconditioning}

\subsection{Level 1 Block Circulant Preconditioning}

Assume $T$ is block Toeplitz with Toeplitz blocks(bttb) with representation 


$$
\begin{aligned}
& T=\left[\begin{array}{ccccc}
T_{0} & T_{-1} & \ldots & T_{2-n_{y}} & T_{1-n_{y}} \\
T_{1} & T_{0} & & & T_{2-n_{y}} \\
\vdots & & \ddots & & \vdots \\
T_{n_{y}-2} & & & T_{0} & T_{-1} \\
T_{n_{y}-1} & T_{n_{y}-2} & \cdots & T_{1} & T_{0}
\end{array}\right] \\
& T_{j}=\left[\begin{array}{ccccc}
t_{0, j} & t_{-1, j} & \cdots & t_{2-n, j} & t_{1-n, j} \\
t_{1, j} & t_{0, j} & & & t_{2-n, j} \\
\vdots & & \ddots & & \vdots \\
t_{n-2, j} & & & t_{0, j} & t_{-1, j} \\
t_{n-1, j} & t_{n-2, j} & \cdots & t_{1, j} & t_{0, j}
\end{array}\right] \\
& L=\left[\begin{array}{ccccc}
L_{0} & -I & O & \cdots & O \\
-I & L_{0} & -I & \cdots & O \\
\vdots & -I & \ddots & \ddots & \vdots \\
O & \ddots & \ddots & L_{0} & -I \\
O & O & \cdots & -I & L_{0}
\end{array}\right] \quad L_{0}=\left[\begin{array}{ccccc}
4 & -1 & 0 & \cdots & 0 \\
-1 & 4 & -1 & \cdots & 0 \\
\vdots & -1 & \ddots & \ddots & \vdots \\
0 & \ddots & \ddots & 4 & -1 \\
0 & 0 & \cdots & -1 & 4
\end{array}\right]
\end{aligned}
$$

where $I$ and $O$ represent $n_{x} \times n_{y}$ identity matrix and zero matrix respectively. We denote $C_{1}(T)$ the level 1 block circulant approximation [1] of $T$. $C_{1}(T)$ is obtained by replacing each of the blocks $T_{j}$ by its best circulant approximation [1] $C_{1}(T)$ and $C_{1}(T)$ is block Toeplitz with circulant blocks. Similarly, one can compute $C_{1}(T)$, the Level 1 approximation to $L$.

Let $A=T * T+\alpha L$, we take $M_{1}(A)$ as a preconditioner for $A$

$$
M_{1}(A)=C_{1}\left(T^{*}\right) C_{1}(T)+\alpha C_{1}(L)
$$

As $C_{1}\left(T_{j}\right)$ is a circulant matrix, it can be expressed as

$$
\mathrm{C}\left(T_{j}\right)=F_{x}^{*} \Lambda_{j} F_{x}, j=1-n_{y}, \ldots, 0, \ldots n_{y}-1
$$

where $F_{x}$ is the $n_{x} \times n_{x}$ Fourier matrix,and $\Lambda_{j}$ is the diagonal matrix whose entries are the eigenvalues of $C\left(T_{j}\right)$. Form this we obtain 


$$
C_{1}(T)=\left(I_{y} \otimes F_{x}\right)^{*} T(\Lambda)\left(I_{y} \otimes F_{x}\right)
$$

Where $I_{y}$ denotes the $n_{y} \times n_{\mathrm{y}}$ identity matrix and $T(\Lambda)$ is the block Toeplitz matrix with the $\Lambda_{j}$ as its diagonal blocks

$$
T(\Lambda)=\left[\begin{array}{cccc}
\Lambda_{0} & \Lambda_{-1} & \cdots & \Lambda_{1-n_{y}} \\
\Lambda_{1} & \Lambda_{0} & \Lambda_{-1} & \vdots \\
\vdots & \ddots & \ddots & \Lambda_{-1} \\
\Lambda_{n_{y}-1} & \cdots & \Lambda_{1} & \Lambda_{0}
\end{array}\right]
$$

There exists a permutation matrix $P$, corresponding to a reindexing of unknowns from column lexicographical order to row lexicographical order, for which $P^{T} T(\Lambda) P$ is a block diagonal. So

$$
C_{1}(T)=\left(I_{y} \otimes F_{x}\right)^{*} P^{T} \operatorname{diag}\left(D_{1}, \cdots, D_{n_{x}}\right) P\left(I_{y} \otimes F_{x}\right)
$$

Where $\left[D_{k}\right]_{i, j}=\left[\Lambda_{i-j}\right]_{k, k}, 1 \leq i, j \leq n_{y}, k=1,2, \cdots, n_{x} . L$ can alse be expressed with a similar form

$$
C_{1}(L)=\left(I_{y} \otimes F_{x}\right)^{*} P^{T} \operatorname{diag}\left(E_{1}, \cdots, E_{n_{x}}\right) P\left(I_{y} \otimes F_{x}\right)
$$

From above representation, we obtained the following expression for the $\mathrm{M}_{1}(A)$

$$
M_{1}(T)=\left(I_{y} \otimes F_{x}\right)^{*} P^{T} \operatorname{diag}\left(D_{k}^{2}+\alpha E_{k}\right) P\left(I_{y} \otimes F_{x}\right)
$$

Now consider the computation of $w=M_{1}(A)^{-1}$,

$$
w=M_{1}(A)^{-1} v=\left(I_{y} \otimes F_{x}\right)^{*} P^{T} \operatorname{diag}\left(D_{k}^{2}+\alpha E_{k}\right)^{-1} P\left(I_{y} \otimes F_{x}\right) v
$$

where $v=\operatorname{vec}(v)$ and $v$ is an $n_{x} \times n_{\mathrm{y}}$ array.

The matrix-vector product $\hat{v}=\left(I_{y} \otimes F_{x}\right) v$ corresponding to applying one-dimensional DFTs to the columns of $v$. The computation $\hat{\mathrm{w}}=P^{T} \operatorname{diag}\left(D_{k}^{2}+\alpha E_{k}\right)^{-1} P \hat{v}$ can be carried out by solving linear systems

$$
\left(D_{k}^{2}+\alpha E_{k}\right) \hat{w}_{k, .}=\hat{v}_{k,,}, k=1,2 \cdots, n .
$$

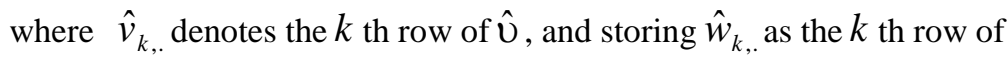
$\hat{\omega}=\operatorname{array}(\hat{w}), \operatorname{array}$ is an inverse operator for $v e c$. Finally, the 
computation $w=\left(I_{y} \otimes F_{x}\right)^{*} \hat{w}$ corresponds to applying inverse DFTs to the columns of $\hat{\omega}$.This yields $\omega=\operatorname{array}(w)$.

\subsection{Level 2 Block Circulant Preconditioning}

We denote the level 2 block circulant approximation to $T$ by $\mathrm{C}_{2}(T) . \mathrm{C}_{2}(T)$ can be obtained by replacing each of the diagonal block matrices $D_{k}$ by its best circulant approximation $C\left(D_{k}\right)$. Let

$$
\left(C_{k}\right) \theta_{y}^{*} \operatorname{dia}\left(\hat{g} l_{k}\right) F_{y}
$$

where $\operatorname{diag}\left(\hat{d}_{k}\right)$ denotes the diagonal matrix whose diagonal entries comprise the components of the vector $\hat{d}_{k} \in C^{n_{y}}$.This yields the representation

$$
\begin{gathered}
C_{2}(T)=\left(I_{y} \otimes F_{x}\right)^{*} P^{T}\left(I_{y} \otimes F_{x}\right)^{*} \times \operatorname{diag}\left(\hat{d}_{1}, \ldots, \hat{d}_{n_{x}}\right)\left(I_{y} \otimes F_{y}\right) P\left(I_{y} \otimes F_{x}\right) \\
=\left(F_{y} \otimes F_{x}\right)^{*} \operatorname{diag}\left(\hat{d}_{1}, \ldots, \hat{d}_{n_{x}}\right)\left(F_{y} \otimes F_{x}\right)
\end{gathered}
$$

Then, we obtain the representation for the $M_{2}(A)$,

$$
\begin{aligned}
& M_{2}(A)=C_{2}(T) * C_{2}(T)+\alpha C_{2}(L) \\
& =\left(F_{y} \otimes F_{x}\right)^{*} \operatorname{diag}\left(\left|\hat{d}_{k}\right|^{2}+\hat{e}_{k}\right)\left(F_{y} \otimes F_{x}\right)
\end{aligned}
$$

The computation $w=M_{2}(A)^{-1} v$ is easy. The matrix-vector product $\hat{\mathrm{v}}=\left(F_{y} \otimes F_{x}\right) \nu$ corresponds to applying the two-dimensional DFT to $v=\operatorname{array}(v)$.Next, to compute $\hat{w}=\operatorname{diag}\left(\left|\hat{d}_{k}\right|^{2}+\hat{e}_{k}\right)^{-1} \hat{v}$, take $\hat{\omega}=\operatorname{array}(\hat{w})$ to consist of columns

$$
\hat{\omega}_{., k}=\hat{v}_{., k} \cdot /\left(\left|\hat{d}_{k}\right|^{2}+\hat{e}_{k}\right) \quad k=1, \ldots, n_{y}
$$

Finally, apply the inverse two-dimensional DFT to $\hat{\omega}$ to obtain $\omega=\operatorname{array}(w)$. 


\section{Simulation and data analysis}

Taking the atmospheric turbulence blur image as an example [7], and the value used for the regularization parameter is $\alpha=0.005$. The image lies on a $256 \times 256$ pixel grid. We apply MATLAB programming to conduct simulation experiment. And then compare the numerical performance of the various circulant preconditioning.

In order to simulate blurred image caused by atmospheric turbulence, we choose $\sigma=0.05$ for point spread function $K(x, y)=\frac{1}{2 \pi \sigma^{2}} e^{-\frac{x^{2}+y}{2 \sigma^{2}}}$, and variance noise is 0.001 . Blurred image $d$ can be obtained from (1) by using multiplication of block Toeplitz matrix with Toeplitz blocks with vector [1]. The original image(a) and blurred image (b) were showed in Figure.1.The restored images without preconditioner were showed in figure.2, (c) is the restored image with CG method iterating 10 times, (d)

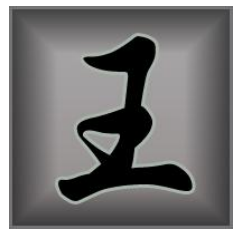

(a)

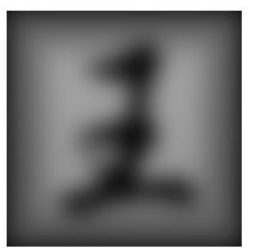

(b)

Fig. 1 The original image (a) and blurred image(b)

is restored image iterating 50 times and (e) is the restored image iterating 100 times. Figure .3 are the restored images with level1 preconditioner, (f) is the restored image with PCG method iterating 10 times, (g) is restored image iterating 50 times and $(\mathrm{h})$ is the restored image iterating 100 times. Figure .4 are the restored images with level 2 preconditioner, (i) is the restored image with PCG method iterating 10 times, $(\mathrm{j})$ is restored image iterating 50 times and $(\mathrm{k})$ is the restored image iterating 100 times. 

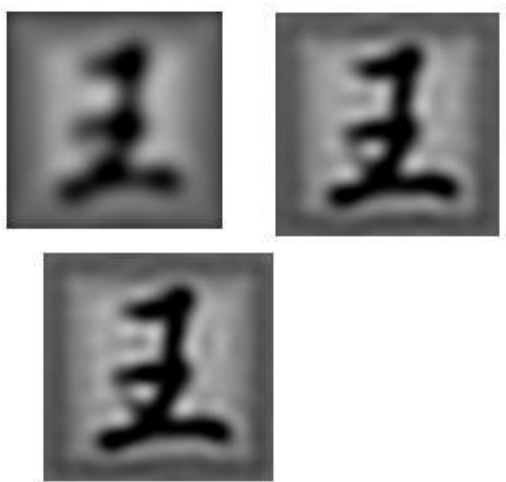

(c)

(d)

(e)

Fig. 2 Restored images without preconditioner

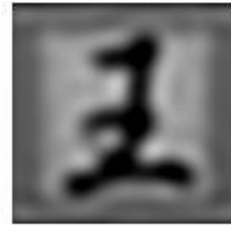

(f)

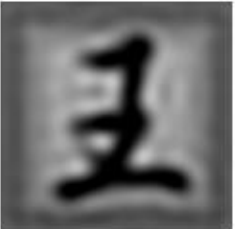

(g)

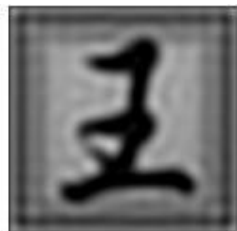

(h)

Fig. 3 Restored images with level1 preconditioner

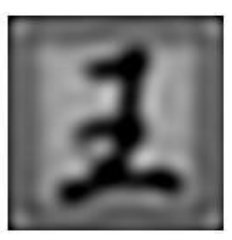

(i)

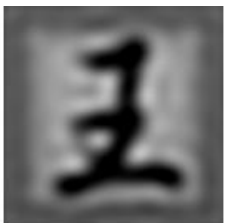

(j)

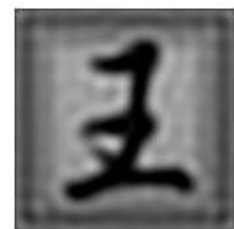

(k)

Fig. 4 Restored images with level2 preconditioner

Table 1 PSNR of restored images with different methods

\begin{tabular}{|l|c|c|c|}
\hline \multirow{2}{*}{$\begin{array}{l}\text { Iteration } \\
\text { times }\end{array}$} & \multicolumn{3}{|c|}{$\begin{array}{c}\text { PSNR of the restored images of } \\
\text { different methods }\end{array}$} \\
\cline { 2 - 4 } & CG & Level1 & Level2 \\
\hline 10 & 17.9596 & 18.1189 & 18.4710 \\
\hline 50 & 18.3075 & 18.5688 & 18.4688 \\
\hline 100 & 17.9077 & 18.6541 & 18.6548 \\
\hline
\end{tabular}


From table.1 we can see that the restored images with PCG methods have higher PSNR than restored images with CG method, and PCG method with level 2 preconditioner is better than other methods.

\section{Acknowledgment}

This work was supported by a grants from the National Natural Science Foundation of China (No. 61071148)

\section{Reference}

1.Vogel .C.R. Computational methods for inverse problems, Montana State University, Bozeman, Montana, 2002.

2.Changfeng Ma. Optimization method and matlab program [M]. Beijing: Science Publisher, 2010.

3.Chen $\mathrm{Pu}$, Tingsong Xiao, Shuming Sun, Mingwu Yuan. Realization of preconditioned conjugate gradient method and improvement [J].Beijing Peking University ("Engineering Mechanics "supplement "). 1998.

4.Strang .G.A proposal for Toeplitz matrix calculations, Studies in Applied Mathematics, 74(1986), pp,171-176.

5.Hanke .M and Nagy .J.G. Toeplitz approximate inverse preconditioner for banded Toeplitz matrices, Numerical Algorithms, 7(1994),pp.183-199.

6.Raymond chan .H, Michael K.N. Conjugate Gradient Methods for Toeplitz Systems, September 1995,TR-CS-95-07.

7.Qingfei Li, Zhichao Zhu, Shuai Fang. Restoration of atmospheric turbulence-degraded image analysis [J], Journal of HeFei University of Technology (NATURAL SCIENCE EDITION), 2011, 34 (1):80-82.

8.Min Yao. Digital image processing [M]. Beijing: Mechanical Industry Press, 2012.4

9.Yanfei Wang. Calculation method of inversion problem and its application [M] ,Beijing: Higher Education Press, 2007

10.Chan.R. Circulant preconditioners for Hermitian Toeplitz systems, SIAM J. Matrix Anal .Appl,10(1989), 542-550.

11. Jain.A.K. Fundamentals of Digital Image Processing, Prentice-Hall, New York, 1989. 